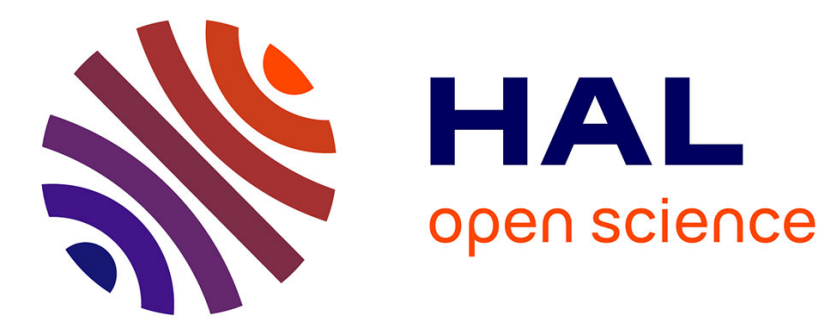

\title{
Facebook, un nouvel outil de campagne : Analyse des réseaux sociaux et marketing politique

\author{
Maria Mercanti-Guérin
}

\section{To cite this version:}

Maria Mercanti-Guérin. Facebook, un nouvel outil de campagne : Analyse des réseaux sociaux et marketing politique. La Revue des Sciences de Gestion, 2010, 242 (2), pp.17. hal-02054422

\section{HAL Id: hal-02054422 \\ https://hal.science/hal-02054422}

Submitted on 4 Mar 2019

HAL is a multi-disciplinary open access archive for the deposit and dissemination of scientific research documents, whether they are published or not. The documents may come from teaching and research institutions in France or abroad, or from public or private research centers.
L'archive ouverte pluridisciplinaire HAL, est destinée au dépôt et à la diffusion de documents scientifiques de niveau recherche, publiés ou non, émanant des établissements d'enseignement et de recherche français ou étrangers, des laboratoires publics ou privés. 


\title{
Analyse des réseaux sociaux et marketing politique Facebook, un nouvel outil de campagne
}

\author{
Maria Mercanti-Guérin \\ Maître de conférences \\ Université d'Evry-Val d'Essonne \\ Centre de recherche DMSP, DRM (CNRS UMR 7088), Université Paris Dauphine
}

Université d'Evry-Val d'Essonne, UFR SSG, 02 rue du Facteur Cheval, 91000 Evry cedex, 


\section{Analyse des réseaux sociaux et marketing politique}

Facebook, un nouvel outil de campagne

Les média ont porté une attention particulière à la façon dont les candidats à la présidentielle américaine 2008 ont intégré Facebook et d'autres sites de réseaux communautaires à leurs stratégies de campagne. Dans ce contexte, la compréhension de la formation et de l'utilisation des réseaux sociaux est d'importance. L'analyse des réseaux sociaux apporte un cadre d'analyse permettant de comprendre comment un réseau social se lance, évolue et peut être utilisé par les candidats. Les outils d'analyse des réseaux sociaux sont utilisés pour visualiser les relations au sein d'un réseau et pour analyser les données d'un réseau en terme de centralité, proximité, liens faibles. Dans cette étude, nous analysons deux échantillons représentatifs de communautés en ligne composées de supporters et d'opposants. Nous détaillons notamment l'impact de la forme des réseaux sur l'engagement des supporters et le partage d'information.

Mots-clé : analyse des réseaux sociaux, sites communautaires, marketing politique

\section{Social network analysis and political marketing}

\section{Facebook, a new campaign tool}

Considerable media attention was focused on how U.S. presidential candidates in 2008 integrated Facebook and other social networking sites into their campaign strategies. In this context, understanding the formation and utilization of online social networks becomes important. Social network analysis provides a framework of understanding how social networks begin, evolve and are utilized by candidates. Network analytic tools are used to represent relationships in a network, and to analyze the network data in term of centrality, closeness, weak ties. In this study we survey two representative samples of online communities of supporters and opponents. We analyze the impact of network design on supporters' involvement and information sharing.

Key-words: social network analysis, social networks, political marketing 


\section{Analyse des réseaux sociaux et marketing politique \\ Facebook, un nouvel outil de campagne}

\section{INTRODUCTION}

La dernière élection présidentielle américaine a récemment mis en lumière l'importance d'Internet et de ses réseaux sociaux dans le succès d'une campagne. Les liens entre Internet et les stratégies des candidats, les enjeux de l'émergence de ce nouveau média dans les discours politiques ont donné lieu à un intérêt nouveau pour le marketing politique. La publicité politique en ligne a représenté un poste budgétaire extrêmement important. Les montants consacrés à l'e-pub par Barack Obama s'élèveraient à plus de cinq millions de dollars ${ }^{1}$. Néanmoins, la publicité en ligne n'aura pas été la seule révélation de cette campagne. En effet, les plate-formes communautaires ont été utilisées pour recruter des partisans, mobiliser des supporters, contrer des rumeurs et décupler l'audience des médias traditionnels ${ }^{2}$. Dans ce contexte, la compréhension du fonctionnement des réseaux sociaux s'enrichit d'une direction supplémentaire : celle de la production d'idées, de la confrontation d'arguments et de la redécouverte de l'électeur comme apporteur de concepts nouveaux en politique. La notion de démocratie participative qui vise à accroître l'implication des citoyens dans le débat public prend tout son sens sur Internet. Elle s'accompagne d'une compréhension du fonctionnement des réseaux. Toutefois, l'appréhension de ce phénomène emprunte aussi bien aux technologies de l'information qu'à la sociologie et plus récemment à la sociométrie. L'analyse des réseaux sociaux ou ARS est une approche sociologique relativement récente. L'objectif de cet article est de décrire à travers les concepts vulgarisés par cette nouvelle discipline assez peu usitée en sciences de gestion comment un réseau se structure et quels peuvent être les indicateurs de sa vitalité. Son application au champ politique permet d'enrichir le marketing politique dans le sens où il définit un nouvel espace public virtuel et donc de nouvelles stratégies de diffusion des idées. Néanmoins un certain nombre de questions reste en suspend. Quelle est la structure des réseaux politiques qui se sont créés sur le Web ? Comment s'articulent entre eux les différents sous-groupes les constituant? Quel type d'information véhiculent-ils ? Quelle est la part de stratégie politique construite et pensée et la part d'aléatoire dans leur création? L'article se compose de trois parties. Dans une

\footnotetext{
${ }^{1}$ Fauconnier F. (2008), La net-campagne de Barack Obama, instrument de sa victoire, Le Journal du Net, 06-112008.

${ }^{2} \mathrm{CNN}$ a signé un partenariat avec Youtube, ABC News avec Facebook.
} 
première partie, nous synthétiserons l'apport de l'analyse des réseaux sociaux dans la compréhension de la circulation des flux d'information ainsi que son importance en marketing et plus spécifiquement en marketing politique. Dans une seconde partie, nous exposerons les résultats de deux recherches fondées sur les groupes politiques créés sur Facebook. Nous mettrons en application les principaux indicateurs de l'ARS mais également proposerons un modèle d'évaluation de la qualité d'un réseau et des grandes mesures constitutives de ses performances. Dans une troisième partie, nous émettrons un certain nombre de suggestions à destination des administrateurs des réseaux politiques et confronterons nos résultats aux enseignements tirés de la littérature portant sur l'ARS.

\section{ANALYSE DES RESEAUX SOCIAUX ET CIRCULATION DES INFORMATIONS AU SEIN DES COMMUNAUTES EN LIGNE, INTERROGATIONS SUR LEUR UTILITE POLITIQUE}

\subsection{Qu'est ce qu'un réseau social?}

\subsubsection{Définition et principales propriétés structurales}

Un réseau social peut se définir comme un ensemble d'individus, organisations ou entités entretenant des relations sociales fondées sur l'amitié, le travail collaboratif, l'échange d'informations...(Garton L. et al., 1997). L'étude des réseaux sociaux est à l'intersection des systèmes d'information, de la sociologie et des mathématiques. De nombreuses recherches empiriques montrent que les comportements individuels sont grandement conditionnés par les réseaux auxquels on appartient. La structure des réseaux constituerait une explication incontournable de certains faits sociaux. Bott E. en 1957 montre que la densité d'un réseau social au sein d'une famille influence le degré de spécialisation des tâches au sein des couples. Par ailleurs, l'étude de la densité des réseaux démontre que la structure a un impact direct sur la circulation de l'information. Granovetter M. (1973) met en avant l'importance des liens faibles dans la diffusion de l'information. Ces derniers circuleraient plus librement qu'au sein d'un réseau présentant une forte cohésion. Ainsi, la chance de trouver du travail ne se ferait pas via l'aide de sa famille mais grâce à des individus avec lesquels l'intimité est moins forte. La centralisation des réseaux est également à prendre en compte dans l'observation des flux de communication. Freeman L.C. (1979) définit un certain nombre d'indicateurs comme le contrôle (centralité d'un membre du réseau par qui passe la majorité de l'information), le degree (nombre d'individus connecté à un individu donné) ou 
l'indépendance (un individu est connecté à un nombre important d'individus ce qui le soustrait de toute dépendance à l'égard d'un seul). Enfin, la notion de proximité a été mise en exergue par Milgram S. (1967). Six degrés de séparation représenteraient le nombre maximum de distance entre deux individus pris de façon aléatoire. Ces travaux ont permis d'associer aux réseaux sociaux la notion d'un monde étroit, un petit monde où les interactions sont possibles entre n'importe quel groupe, communauté ou culture. Au concept de monde étroit s'ajoute l'idée de réseaux limités en taille. La règle de 150 ou nombre de Dunbar issue de l'anthropologie et de la psychologie évolutionniste fixe une limite humaine à la taille maximale d'un réseau (150). Au-delà, la reconnaissance des membres du groupe ne serait plus assurée.

L'émergence des sites communautaires apporte des applications pratiques à ces théories, des développements futurs mais également des remises en causes. Cai D. et al. (2006) notent qu'un réseau ne peut être analysé seul et de façon indépendante. L'analyse doit être contextuelle, les réseaux s'influençant mutuellement. Sing S. (2006) cite les efforts de Google Lab pour fédérer à travers une plate-forme unique des réseaux communautaires différents. L'émergence de méta-réseaux n'a pas été encore étudiée.

\subsubsection{Réseau social, sociabilité et amitié}

Si les frontières des réseaux posent question, les qualificatifs appliqués aux liens le sont également. Les liens forts seraient un facteur de confiance important dans un réseau. L'affiliation de mes amis intimes à un réseau m'inciterait à adhérer à ce réseau dans un processus enfermant qui m'éloignerait des ressources apportées par les liens faibles. Les réseaux communautaires seraient donc des répliques de réseaux sociaux leur préexistant (réseaux de la vie réelle versus réseaux de la vie virtuelle). Néanmoins, de nombreux auteurs soulignent que la notion d'amis est inappropriée à l'amitié telle qu'elle se pratique dans la vie réelle (Lelong B., Thomas F., 2001). Le nombre important « d'amis » référencés sur les pages de Facebook remet en cause la règle des 150 puisque beaucoup d'internautes assimilent nombre d'amis à popularité. Enfin, comment définir dans ce cadre un lien faible ? Est-ce une personne dont la connaissance est purement virtuelle? Haythornthwaite C. (2005) parle de liens latents pour qualifier ces nouvelles formes de relation. Par ailleurs, l'intensité de la sociabilité varierait avec le temps et le niveau de vie, les plus jeunes et les catégories socioprofessionnelles supérieures auraient une sociabilité plus intense et plus tournée vers l'extérieur que les plus âgés et les catégories inférieures (Rivière C. , 2000). Ces constatations 
expliqueraient le succès des réseaux sociaux sur certaines cibles. Parallèlement se développeraient des formes substituables de sociabilité qui combleraient le déclin observé de ce que l'on peut nommer «le temps traditionnel de sociabilité ${ }^{3} »$. La sociabilité téléphonique (grâce au recours croissant aux SMS notamment) est en pleine croissance (Rivière C., 2000). Elle fait écho à celle développée via les courriers électroniques (Lelong B., Thomas F., 2001). A cette modification des supports de sociabilité s'ajoute un changement dans les modes de sociabilité.

\subsubsection{La sociabilité du clair obscur ${ }^{4}$ : vers un bouleversement du principe d'homophilie?}

Le clair-obscur est un mode de visibilité de l'identité propre à certaines plateformes relationnelles comme Facebook. Les individus «profitent de la plasticité de la visibilité sur Internet pour constituer des cercles relationnels avec des proches qu'ils connaissent et rencontrent souvent dans la vie réelle tout en restant relativement cachés des autres » (Cardon D., 2008, p 110). Les réseaux amicaux se construisent alors sur des connaissances préalables faites dans la vie réelle. Ils s'étendent à des inconnus lorsque ceux-ci peuvent appartenir à la zone relationnelle préalablement définie (mêmes école, ville, université). Néanmoins, les éléments les plus actifs substituent une logique relationnelle à une logique purement identitaire, fondée sur le partage de contenus numériques et l'adhésion à des groupes (dont les groupes politiques). Ils poursuivent une logique d'accumulation au centre de l'identité numérique et du capital réputationnel. L'obscur appartient alors au relationnel intime et aux liens forts alors que le clair s'exprime dans des liens faibles et au sein de groupes à l'homophilie plus que distendue. Pourtant c'est sur le principe même d'homophilie, de ciblage de groupes cohérents en terme de consommation que se construisent les principales techniques liées au marketing des réseaux dont le marketing viral.

\subsection{Réseau social et marketing : la viralité à l'honneur}

Dans le champ du marketing, l'étude des réseaux et les implications et perspectives nouvelles qu'elle offre sont infinies (Iacobucci D., 2007). L'analyse des réseaux ne touche pas uniquement le consommateur et les processus d'adoption des innovations mais également les

\footnotetext{
${ }^{3}$ Putnam R.D.(2000) montre que la sociabilité baisse de manière drastique depuis 30 ans aux Etats-Unis entraînant un affaiblissement de la participation politique, civique, religieuse et syndicale.

${ }^{4}$ Le modèle du clair-obscur est un modèle de visibilité de l'identité sur le Web 2.0 décrit par Cardon D. (2008).
} 
relations inter-organisations (clients, fournisseurs, concurrents). Par ailleurs, de vraies réflexions sont engagées et encore renforcées grâce au Web 2.0 sur les concepts d'influenceurs, «recommandeurs », adopteurs précoces en un sens les initiateurs, administrateurs et leaders des réseaux. Enfin, l'analyse des réseaux apporte de nouvelles conceptualisations et mesures à des phénomènes anciens comme le bouche à oreille remis au goût du jour par Internet (Chabi S., 2008) via le Buzz, marketing viral, ou l'Internet wom 5 . Les communautés de marque sont observées in vivo grâce à l'ethnographie et les données de conversation initialement privées représentent un champ d'analyses sémantiques accessible (Godes D. et Mayzlin D., 2004). La compréhension des phénomènes de diffusion prend en compte de façon accrue les contextes d'interaction et de ce fait les techniques de ciblage pratiquées.

\subsubsection{Homophilie et clustering : le marketing à la recherche des petits mondes}

L'analyse structurale des réseaux met en exergue la notion d'homophilie qui est déterminante dans la réflexion marketing. Elle ne peut se réduire à une représentation graphique des relations entre individus et illustre à travers la visualisation des liens ou nœuds entre individus (également nommés sommets) la notion de proximité :

A travers la distance géodésique entre deux sommets (distance la plus courte séparant deux individus)

A travers son coefficient de clustering, probabilité pour un sommet $\mathrm{v}$ que ses deux voisins v1 et v 2 soient également voisins entre eux

Il apparaît que la majorité des graphes existant dans notre environnement présente trois caractéristiques principales : une faible distance géodésique, un fort coefficient de clustering et une distribution particulière des degree à savoir une grande majorité de sommets à faible degree et un nombre faible de sommets de très hauts degree. Comme le précise Chabi S. (2008), ces sommets ou Hubs sont « ces leaders qui rendent le monde petit » (Chabi S., 2008, p 98). En effet, par ces Hubs ou leaders d'opinion par lesquels passe la majorité des contacts, le réseau prend une forme resserrée, celui « d'un petit monde ».

\footnotetext{
${ }^{5}$ Référence à word of mouse au lieu de word of mouth (bouche à oreille)
} 
Toutefois, Steyer A. et al. (2007) montre que les réseaux sans échelle $\quad{ }^{6}$ (scale free network) suivent des lois de puissance peu prévisibles dans le sens où elles ne sont pas gaussiennes. Ainsi, la gestion des leaders d'opinion (voire même la reconnaissance de leur importance) et d'une façon plus générale les connaissances sur la diffusion de l'information au sein de groupes de discussion en ligne sont largement remises en cause. «Le manager doit donc s'attendre à tout moment à des surprises, dans un sens ou dans l'autre, diffusion très rapide de l'information ou arrêt brutal » (Steyer A. et al., 2007, p 39).

\subsubsection{Réseaux sociaux et marketing politique: de la construction à la déconstruction des} collectifs

Alors que le marketing viral (et, ce malgré certaines recherches comme celle mentionnée cidessus) continue à s'appuyer sur des techniques coûteuses de ciblage des leaders d'opinion, la littérature consacrée au marketing politique pointe la difficulté d'appréhender ces « réseaux mouvants » et n'a pas encore de réflexion vraiment construite sur les leaders d'opinion qui animent ces réseaux ainsi que sur la viralité des informations mises en ligne. Les sites communautaires constituent un réservoir non négligeable de partisans. Néanmoins, la faiblesse ou latence des liens entre ses membres rendrait difficile le contrôle et l'implication idéologique (Bennett W.L., 2003). Plus encore, la notion de capital social définie comme un ensemble de valeurs collectives qui pousse à l'engagement ne serait plus aussi présente au sein des groupes pratiquant la politique en ligne. Bennett W.L. (2003) suggère que la faiblesse organisationnelle de certaines communautés en ligne est adaptée à de nouvelles formes politiques moins centralisées et plus perméables. Bimber B. (1998) cité par Williams C.B. et Gulati G.J. (2006) prédit la remise en cause de l'influence des élites et des groupes organisés. Au-delà de ces interrogations face à un phénomène relativement récent dans nos démocraties, l'élection du dernier président américain illustre l'importance des réseaux sociaux dans la conquête du pouvoir. Williams C.G. et Gulati G.J. (2006) quantifient l'effet positif de Facebook sur les votes ${ }^{7}$ ce qui conforte les décideurs politiques dans leur utilisation de plus en plus professionnelle des réseaux sociaux. La politique 2.0 se caractérise par :

Une présentation du candidat plus authentique et accessible

Une plate-forme d'idées, de réactions, un observatoire de l'opinion

\footnotetext{
${ }^{6}$ Les réseaux sans échelle sont définis comme des réseaux dans lesquels certains nœuds ont un nombre de connections important alors que d'autres en ont peu (Chabi S., 2008). Ils ont été introduits par Barabasi A. et Albert R.A. (1999).

${ }^{7}$ Un nombre de soutiens deux fois supérieurs correspondrait à $1.1 \%$ de voix supplémentaires.
} 
Un catalyseur de l'engouement pour un candidat

Une remise en cause des structures d'autorité et de hiérarchie (groupes spontanés, décentralisation, auto-organisation)

Une réintégration de certaines couches sociales (jeunes ${ }^{8}$ ) dans le débat démocratique

Un nouvel outil de marketing relationnel ${ }^{9}$ et de récolte de fonds

Un palliatif du déclin des structures associatives, traditionnel relais local des politiques

Un support pour les actions locales et l'évènementiel

Deux tendances contradictoires émergent de cette implication croissante des réseaux dans la politique. D'une part, il existe une réelle spontanéité et liberté dans la création d'un réseau, ce qui paradoxalement dépossède le candidat de la maîtrise de sa communication. D'autre part, se construit peu à peu une réelle expertise et professionnalisation de l'utilisation des réseaux et du lien social à travers une communication push qui se veut non invasive. Paradoxalement, cette professionnalisation peut entraîner une désaffection des internautes pour le candidat. Le profil participatif et sociétal de ce nouvel outil nécessite d'en comprendre les codes et les usages. De ce fait, son pilotage est rendu délicat. En outre, l'émergence de groupes hostiles est pratiquement impossible à contenir ${ }^{10}$.

\subsubsection{Politique en ligne : quelle modélisation de la production des internautes dans le débat} politique?

Greffet F. et Wojcik S. (2008) dans un article de synthèse mettent en exergue la difficulté des chercheurs à conceptualiser d'une manière propre à la communication en ligne le débat politique. Pour certains, la discussion électronique a une influence directe sur l'engagement ultérieur des internautes (Monnoyer-Smith L., 2006). Greffet F. et Wojcik S. (2008) soulignent que la sociologie des communautés en ligne amène à s'interroger sur la notion même de délibération et de participation active des internautes au débat public. La difficulté d'opérationnaliser le concept de délibération pose un certain nombre de questions théoriques

\footnotetext{
${ }^{8}$ «Students for Barack Obama » a été créée officiellement de façon spontanée par une étudiante de 21 ans. En quelques mois, ce groupe a compté des dizaines de milliers de membres et des comités dans plus de 80 universités.

9 Joe Trippi, organisateur de l'e-campagne d'Howard Dean en 2004 souligne que « il faut six mois pour créer une mailing-list de 139000 personnes contre 15 jours sur Facebook.» (Netpolitique, www.netpolitique.net/php/interviews/interview33.php3

). Les posts (billets laissés par les amis sur le site) sont également source de viralité plus forte qu'un classique et impersonnel e-mail.

${ }^{10}$ Nous l'appellerons le phénomène « One Million », l'objectif étant pour un groupe anti d'atteindre un million de membres contre un candidat. Un des plus célèbres est le groupe anti- Hillary (One Million Strong against Hillary) qui regroupe près de 400000 membres.
} 
et méthodologiques concernant la mesure tant qualitative que quantitative de la participation politique. Ainsi, Dumoulin M. (2004) souligne que les échanges électroniques ressemblent à « des monologues interactifs » sans rencontre et accord des participants, en un mot sans débat. Par ailleurs, l'évaluation de la qualité des échanges se heurte à une forte subjectivité et à un certain nombre d'essais de modélisation assez contestables. Nous citerons Schneider S.M. (1996) qui s’appuie sur quatre critères (égalité, diversité, réciprocité et qualité des échanges) ou Dahlberg L. (2001) sur six critères (rationalité, réflexivité, empathie, sincérité, inclusion, équité discursive et autonomie). Enfin, la notion d'interaction et de contextualisation des discussions en un mot l'influence du support sur la participation est très peu étudiée. Les recherches dédiées à l'influence d'Internet sur la participation politique mettent surtout en exergue le caractère désinhibé (et donc souvent violent et négatif) des avis des participants. L'hostilité, le conflit, la production de messages brefs et agressifs seraient une des constantes de la communication politique en ligne (Matuszak C., 2007).

\section{CADRE CONCEPTUEL DE RECHERCHE ET METHODOLOGIE}

\subsection{Cadre conceptuel}

Au travers de la littérature, se posent donc deux questions centrales :

1. Quelles sont les caractéristiques d'un réseau politique en ligne ? Est-il fondé sur des liens forts (la conviction politique assurant à elle seule une certaine homophilie et donc une forte cohérence du réseau) ou au contraire des liens faibles? Dans ce cas, sur quel type de groupe s'appuyer pour influer sur la forme du réseau et sa croissance?

2. Quels sont les indicateurs de la qualité d'un réseau politique en ligne? Comment mesurer la participation active de façon contextuelle (c'est-à-dire adaptée aux caractéristiques d'un réseau social virtuel), qu'elle soit positive ou négative ?

La conjugaison de ces trois variables détermine un profil de réseau spécifique. 


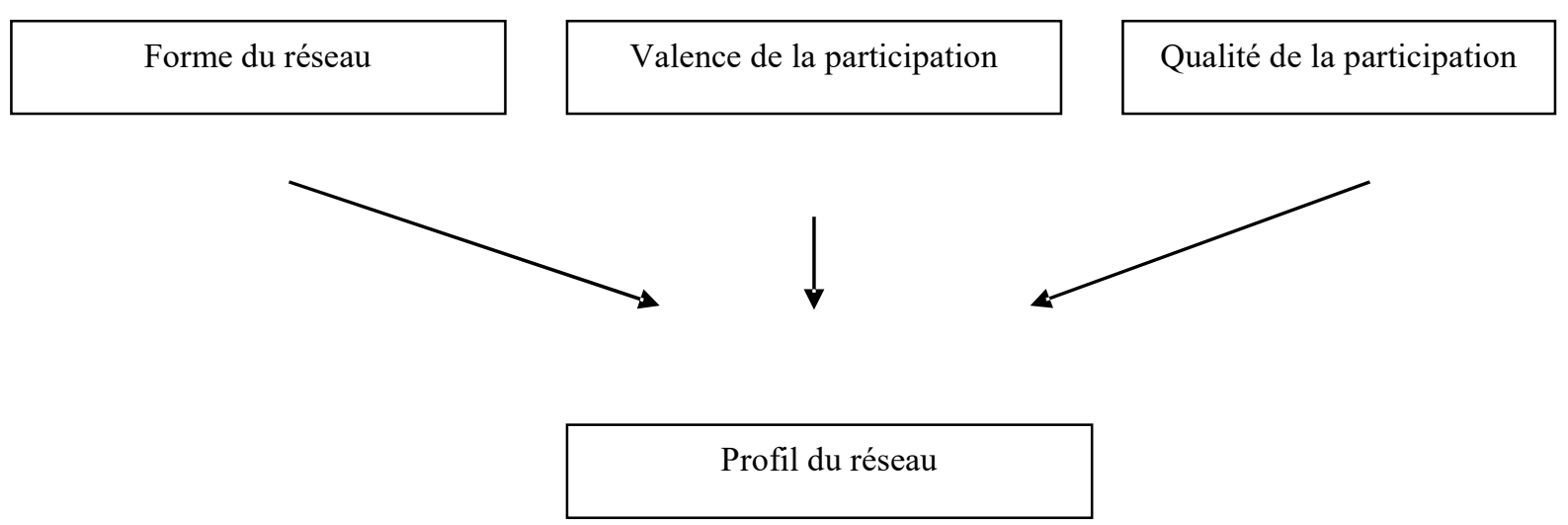

\section{Schéma 1 : Cadre conceptuel de recherche}

\subsection{Opérationnalisation des variables}

La forme d'un réseau peut s'apprécier sur trois niveaux :

Le niveau structural : identification de la structure de l'ensemble et des sousensembles d'acteurs à l'intérieur du système

$\S$ Le niveau relationnel : étude des sous-ensembles qui peuvent être reconstitués à partir d'une mesure de la cohésion ou de la densité des relations entre acteurs

$\S$ Le niveau individuel : analyse des mesures de centralité ou de prestige d'un acteur (groupe) mais également la capacité de diffusion du groupe ( taille du groupe, nombre d'amis de l'administrateur principal, nombre de groupes auxquels l'administrateur appartient, groupes associés).

La qualité de la participation est liée à la production de contenus mis en ligne sous toutes ses formes. Cette variable doit être contextualisée c'est-à-dire adaptée à la production de données telle qu'elle existe sur Facebook. En nous fondant sur une typologie des fonctionnalités des sites Internet, il apparaît que trois types de fonctionnalité peuvent être distingués (Rechenman, 2001):

$\S$ Les fonctionnalités informationnelles concernent les sites émetteurs d'informations quel que soit leur contenu.

$\S$ Les fonctionnalités relationnelles signifient que le site diffuse de l'information mais a également mis en place des fonctionnalités de recueil des avis et de traitement des demandes d'information.

$\S$ Les fonctionnalités transactionnelles sont orientées sur la vente en ligne. 
Dans le cadre des réseaux sociaux, les fonctionnalités transactionnelles sont très peu développées voire inexistantes ${ }^{11}$.

Nous distinguerons donc quatre indicateurs pouvant servir d'évaluation de la qualité de la participation au sein d'un groupe.

${ }^{11}$ Sur Facebook, très peu d'applications sont monétarisées, le modèle économique reposant essentiellement sur la publicité. 


\begin{tabular}{|l|l|l|}
\hline Capacité informationnelle & $\begin{array}{l}\text { Fonctionnalité } \\
\text { informationnelle }\end{array}$ & $\begin{array}{l}\text { Nombre d'événements publiés, } \\
\text { nombre d'éléments publiés }\end{array}$ \\
\hline Indicateur de production & $\begin{array}{l}\text { Fonctionnalité } \\
\text { relationnelle }\end{array}$ & $\begin{array}{l}\text { Nombre de forums de } \\
\text { discussion }\end{array}$ \\
\hline Richesse multimédia & $\begin{array}{l}\text { Fonctionnalité } \\
\text { informationnelle }\end{array}$ & $\begin{array}{l}\text { Nombre de photos et vidéos } \\
\text { publiées }\end{array}$ \\
\hline Indicateur d'interactivité & $\begin{array}{l}\text { Fonctionnalité } \\
\text { relationnelle }\end{array}$ & $\begin{array}{l}\text { Nombre de participants aux } \\
\text { forums de discussion, nombre } \\
\text { de messages mur }\end{array}$ \\
\hline
\end{tabular}

Tableau 1 : indicateurs de production et de diffusion de connaissance au sein des groupes

La valence de la participation peut s'apprécier, quant à elle, par le nombre de groupes hostiles constituant le réseau et le niveau de participation enregistré en leur sein.

\subsection{Méthodologie et descriptif du terrain de recherche}

Le terrain de recherche est axé sur un réseau social considéré à l'heure actuelle comme un des plus dynamiques. Facebook estime le nombre de ses utilisateurs à pratiquement 7 millions de membres en février 2009. Près de 30\% des utilisateurs se situeraient dans la tranche d'âge 1824 ans avec 20 minutes passées en moyenne sur le service par jour.

La méthodologie adoptée est une méthodologie de type netnographique. La netnographie peut se définir comme l'ethnographie appliquée à Internet. L'objectif est d'observer la nature et l'intensité des interactions d'une communauté en ligne sans y participer. Füller J. et al. (2008) identifie cinq étapes structurant la méthodologie de la netnographie :

$\S$ Etape 1: Détermination des objectifs de la recherche et du profil idéal des membres de la communauté

$\S$ Etape 2 : Identification et sélection des communautés

$\S$ Etape 3 : Observation et collecte des données

$\S$ Etape 4 : Analyse des données et interprétation des résultats

$\S$ Etape 5 : Transfert des résultats en solution.

Le contexte de l'étude est celui du congrès de Reims du 14 au 16 novembre 2008 destiné à élire un nouveau premier secrétaire au Parti Socialiste. A cette occasion trois motions se sont dégagées à l'issue du premier tour : la motion C de Benoît Hamon ; la motion D de Martine Aubry et la motion E menée par Ségolène Royal. Le débat d'idées et les contestations qui ont suivi se sont déroulées par média interposés mais ont également pris une tournure particulièrement active au sein des blogs et des communautés en ligne. Les réseaux sociaux 
comme Facebook sont progressivement investis par les politiques français ${ }^{12}$. Deux terrains de recherche distincts ont été sélectionnés. Le premier terrain a été consacré à l'étude d'un réseau et de ses composants : effets d'interactions, centralité de certains groupes, structure. Nous avons choisi le réseau de Benoît Hamon qui est un réseau de taille moyenne (59 groupes référencés sur Facebook) permettant une première analyse sur le voisinage et la centralité des groupes au sein de ce réseau. Le second terrain de recherche porte sur 70 groupes faisant référence aux candidates Aubry et Royal. Cette seconde étude illustre plus spécifiquement la production des connaissances, la construction et le partage des idées au sein des communautés virtuelles. L'analyse netnographique d'un groupe Facebook est détaillée dans la figure 1. Le recueil des données s'est effectué sur une période d'un mois allant de mi-novembre à midécembre 2008. Le traitement des données a nécessité de s'appuyer sur plusieurs méthodes statistiques. La première méthode, l'ARS nous a permis d'illustrer la démarche de profilage d'un réseau en fonction des trois niveaux d'analyse décrits précédemment (structural, relationnel, individuel). La mesure de la valence de la participation et la qualité de participation ont été étudiées en utilisant les méthodes dites classiques d'analyse de données (tests de moyenne, analyse en composantes principales). Le recours à plusieurs méthodes de traitement de données complétant l'ARS est mentionné dans de nombreuses recherches. Lazega E. (1998, p 113) insiste sur « la nécessité de combiner une approche structurale avec d'autres méthodes ».

\section{RESULTATS DE L'ETUDE EMPIRIQUE}

\subsection{Structure d'un réseau politique virtuel : du politisé à l'apolitique}

Le réseau de Benoît Hamon était constitué en Novembre 2008 de 59 groupes. Ces groupes étaient divisés en deux réseaux : Un premier réseau que nous qualifierons de réseau premier à savoir les groupes référencés par le moteur de Facebook et dont la raison d'être est le support ou au contraire l'opposition à Benoît Hamon, le nom du candidat étant systématiquement cité dans la description du groupe. Un deuxième réseau que nous qualifierons de secondaire constitué des groupes dits associés.

\footnotetext{
12 « La semaine dernière, l'UMP a été le premier parti français à se lancer officiellement sur Facebook en créant un groupe "UMP-Fédération numérique" qui a attiré en quelques jours près de 900 membres. Facebook est devenu un "outil incontournable pour toute une génération de militants", a estimé Thierry Solère, secrétaire national de l'UMP en charge des questions Internet ». Source : AFP, 21/10/2007
} 


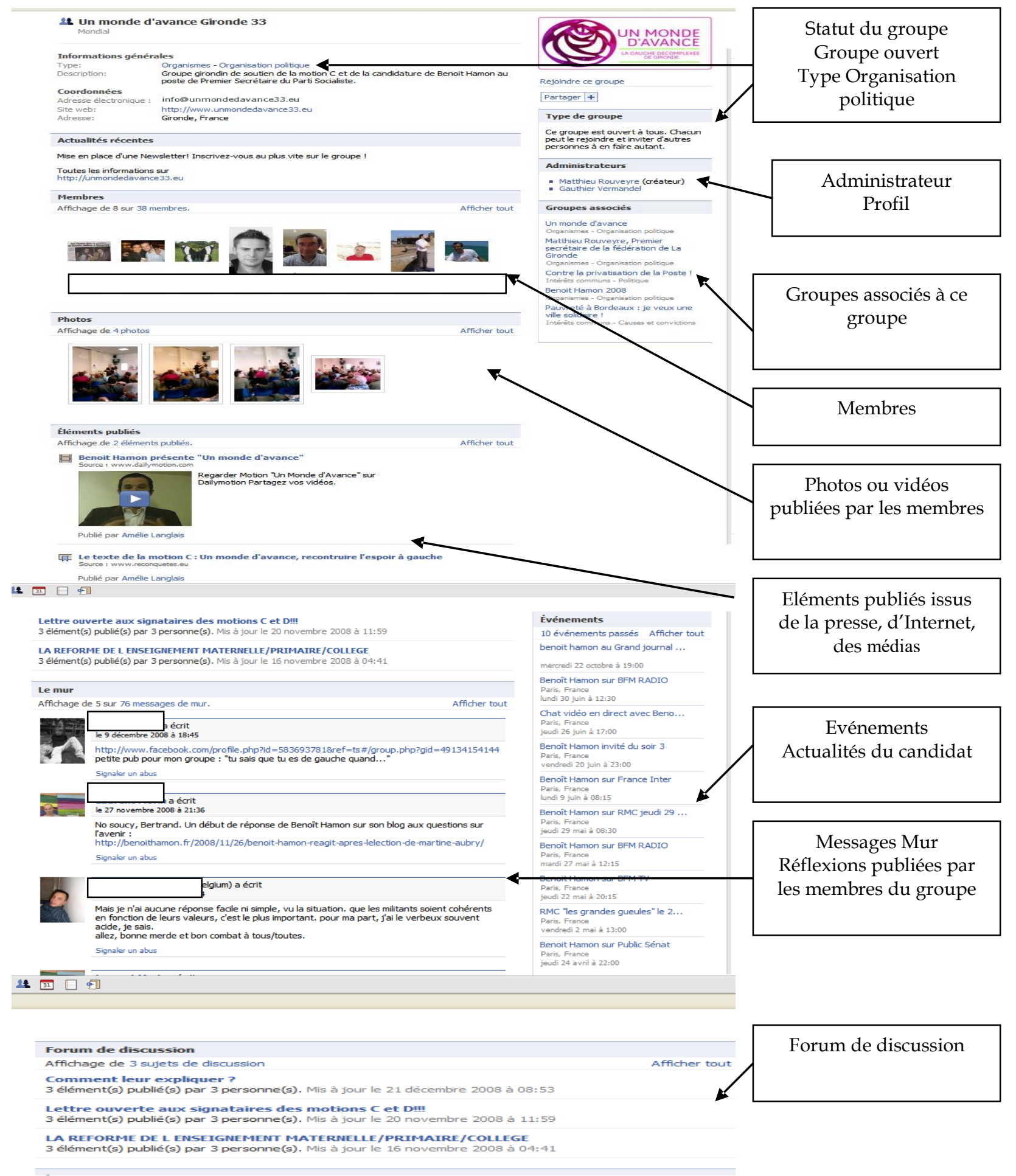

\section{Figure 1 : analyse netnographique d'un groupe sur Facebook}

Leur raison d'être n'est pas Benoît Hamon mais il existe soit un lien univoque entre un groupe premier et ce groupe, soit un lien mutuel. L'analyse du réseau permet d'établir une cartographie (Schéma 2) ou graphe correspondant à un ensemble de raccordement entre plusieurs groupes (dits aussi nœuds). Un lien entre deux groupes est qualifié de dyade. Les groupes étudiés ont été classifiés selon les rubriques proposées par Facebook. Chaque administrateur choisit pour son groupe une catégorie d'appartenance ou type (c.f. légende 
schéma 2). Ainsi, les groupes politiques peuvent être classés, soit dans «Intérêts communs, politique », soit dans "Organismes et associations politiques ». Les groupes classés dans «Organismes et associations politiques » émanent de fédérations ou de groupes politiques identifiés (exemple les jeunes du PS). Les groupes appartenant à la catégorie «Intérêts communs, politique » sont souvent créés par des citoyens plus indépendants des structures politiques établies. Les groupes classés dans la rubrique «Intérêts communs, juste pour le plaisir » sont des groupes anecdotiques ou parodiques, éloignés des préoccupations politiques.

\begin{tabular}{|l|l|}
\hline Nombre de groupes observés & 59 \\
\hline Nombre de dyades & 135 \\
\hline $\begin{array}{l}\text { Nombre de dyades Groupes politiques - } \\
\text { Groupes }\end{array}$ & 59 \\
\hline $\begin{array}{l}\text { Nombre de dyades Groupes Arts, Sports et } \\
\text { Loisirs - Groupes }\end{array}$ & 10 \\
\hline $\begin{array}{l}\text { Nombre de dyades Groupes Intérêts } \\
\text { communs, juste pour le plaisir - Groupes }\end{array}$ & 16 \\
\hline $\begin{array}{l}\text { Nombre de dyades Groupes Intérêts } \\
\text { communs, causes et convictions - Groupes }\end{array}$ & 13 \\
\hline $\begin{array}{l}\text { Nombre de dyades Groupes Intérêts } \\
\text { communs, politique - Groupes }\end{array}$ & $: 13$ \\
\hline
\end{tabular}

Tableau 2 : description du réseau

Ainsi, il apparaît que le réseau politique décrit ne se fonde pas uniquement sur les convictions politiques mais regroupe des motivations très diverses (qui vont des causes sociales au divertissement). Cette diversité est un signe (même si il faudrait le confirmer par d'autres analyses) d'une homophilie relativement faible. La structure du réseau tendrait alors à montrer que la distance géodésique et le coefficient de clustering des différents groupes sont forte pour la distance et faible pour le clustering.

\subsection{Centralisation du réseau et forme : l'importance des liens faibles}

Le degré de centralisation d'un groupe ou nœud se réfère au nombre de nœuds qui lui sont connectés. Dans le cas de la cartographie des groupes Facebook de Benoît Hamon, la centralité la plus forte est observée chez les groupes suivants « Benoît Hamon 2008 » et «Un monde d'avance » ce qui conforte le profil relativement politique de cette cartographie. 


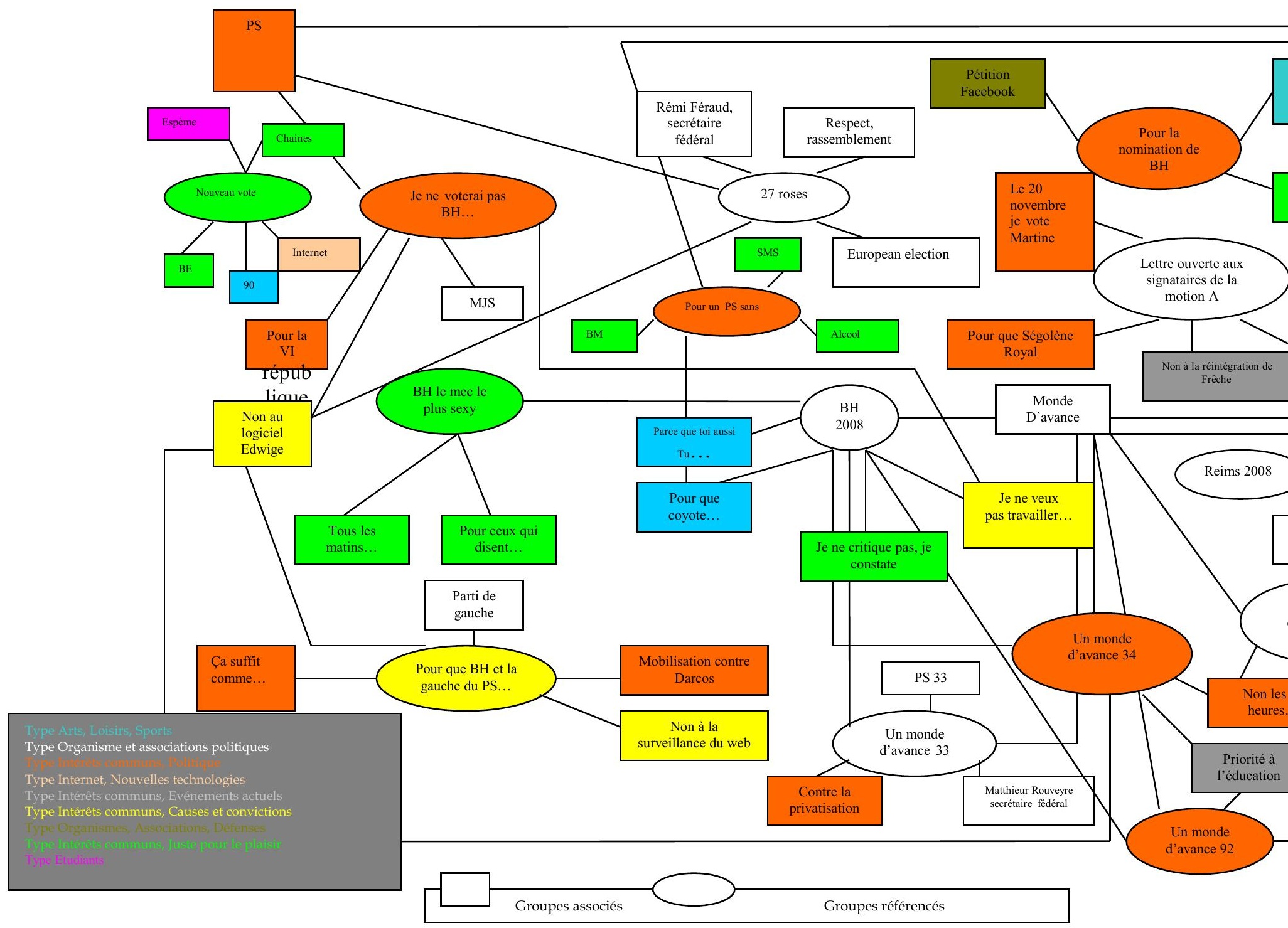

Schéma 2 : Cartographie des groupes liés au candidat Benoît Hamon 
Par contre, le rapport des liens du groupe «Benoît Hamon 2008 » sur le total des liens du réseau est relativement faible (0.07) ce qui indique peu de centralisation. Le contrôle se réfère à la centralité d'un groupe. Celui-ci est dit central pour le réseau si il sert de point de communication entre les groupes. Ainsi, un groupe central est un pont entre les différents groupes constituant le réseau. Parmi les groupes dits associés, nous pouvons noter l'importance de deux groupes appartenant à la catégorie Intérêts communs, causes et convictions à savoir «Non au logiciel Edwige» et «Je ne veux pas travailler jusqu'à 70 ans ». Ces groupes servent de diffuseurs d'informations vers d'autres groupes soit politiques, soit appartenant à d'autres catégories plus éloignées comme la catégorie «Juste pour le plaisir ». Par ailleurs, la forme générale du réseau est également à prendre en compte. Kumar R., Novak J. et Tomkins A. (2006) à travers leur analyse de deux groupes de réseaux sociaux, Flickr et Yahoo 360 montrent que l'activité d'un réseau est dépendant dans son développement de trois types de liens :

$\S$ Les singles n'ont aucune connexion mais sont néanmoins importants. Le groupe « Reims $2008 »$ n'entretient aucune connexion avec les autres groupes. Il est mentionné par Facebook comme faisant référence à Benoît Hamon mais également aux autres candidats puisque c'est un groupe de discussion des motions. Il regroupe 117 membres.

$\S$ Les grands composants sont des sous-réseaux regroupant un nombre de groupes fortement reliés entre eux. Ainsi, la nébuleuse «Un monde d'avance » et ses déclinaisons locales «Un monde d'avance 34, 78,92...» constituent un grand composant.

$\S$ Les régions moyennes représentent des groupes isolés qui interagissent entre eux sans lien avec le reste du réseau. « Lettre ouverte aux signataires de la motion A » dispose d'un seul lien avec un groupe associé («PS ») le rattachant au réseau.

Kumar R., Novak J. et Tomkins A. (2006) démontrent que plus de la moitié d'un réseau se structure en dehors des grands composants. Les régions moyennes sont de mini-réseaux sociaux, généralement créés par des administrateurs dynamiques et représenteraient dix pourcents des utilisateurs de Yahoo 360 !. Elles seraient déterminantes dans la croissance d'un réseau car elles rejoignent graduellement les grands composants qu'elles contribuent à enrichir. Afin de vérifier la « vitalité » de ces zones, nous avons étudié l'interactivité au sein des groupes présentant plus de deux liens et ceux présentant un seul lien. Il apparaît que l'interactivité est plus forte au sein des groupes fortement reliés entre eux. Néanmoins les courbes de distribution et l'analyse des coefficients de symétrie et d'aplatissement montrent 
que les groupes faiblement reliés présentent une distribution de l'interactivité non normale. En effet, certains groupes sont fortement interactifs en leur sein ce qui tendrait à confirmer l'existence d'un profil particulier de groupes faiblement interreliés et très interactifs.

\begin{tabular}{|l|l|}
\hline Groupes présentant un seul lien & 30 \\
\hline $\begin{array}{l}\text { Moyenne de l'interactivité au sein des } \\
\text { groupes }\end{array}$ & 119 \\
\hline Groupes présentant deux liens et plus & 29 \\
\hline $\begin{array}{l}\text { Moyenne de l'interactivité au sein des } \\
\text { groupes }\end{array}$ & 211 \\
\hline
\end{tabular}

Tableau 3 : Moyenne de l'interactivité au sein des groupes faiblement et fortement reliés
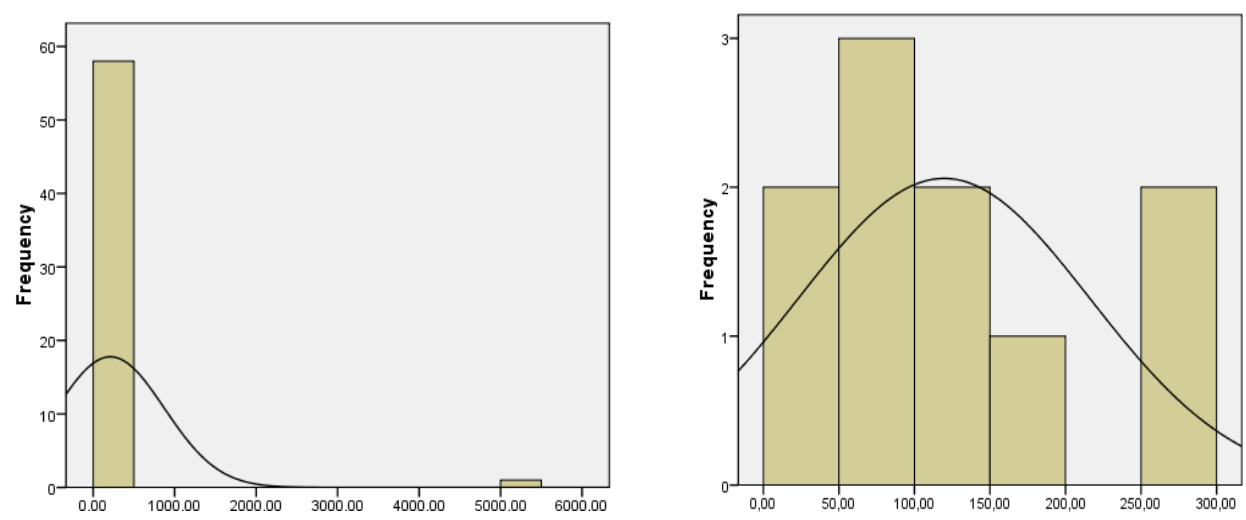

Schéma 3 : Distribution de fréquences de l'interactivité groupes fortement reliés (courbe 1) versus faiblement reliés (courbe 2)

\subsection{Les « groupes ponts politiques » faiblement « diffuseurs » d’information}

Afin d'analyser la qualité des interactions des groupes constituant le réseau de Benoît Hamon, nous avons concentré notre analyse sur les groupes politiques (intérêt commun -politique et groupes politiques) de son réseau. Nous avons travaillé avec le logiciel Netminer3 à partir d'une matrice dite de proximité dite aussi matrice de données relationnelles (Calvani A. et al., 2005). Mazzoni E. (2006) définit les données relationnelles comme des interactions entre sujets. Ces interactions sont représentées par l'intersection entre lignes et colonnes qui permet de visualiser les liens entre individus d'un même réseau (notées 1 ou 0 ). 


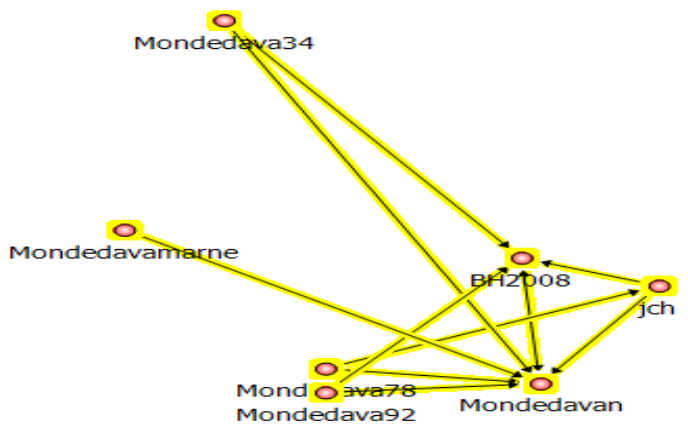

\section{Schéma 4 : Représentation graphique ou sociogramme du réseau politique favorable à}

\section{Benoît Hamon}

La qualité des relations au sein du réseau peut être mesurée selon plusieurs indicateurs. Le premier d'entre eux est l'indicateur de densité compris entre 0 et 1 . Un indicateur à 1 illustre une situation optimale de relations. Dans le cas du réseau étudié, l'indicateur de densité est de 0.262 ce qui illustre, soit une faible densité des interactions entre groupes, soit une fort pourcentage d'exclusions (nombre de groupes n'interagissant pas avec le reste du réseau). En ce qui concerne notre réseau d'étude, la faiblesse de l'indicateur de densité est essentiellement due à la faiblesse des relations réciproques au sein du réseau.

En effet, selon Mazzoni E. (2006), l'indicateur de densité est fondé sur :

$\S$ L'indicateur d'inclusion qui évalue le pourcentage de sujets participant aux échanges du groupe. Dans notre sélection, l'inclusion est à 100\% (c.f. tableau 4) ce qui montre que l'ensemble des groupes étudiés fait référence à un au moins des groupes de l'échantillon ou est cité par un au moins des groupes de l'échantillon.

$\S$ Le Degree des sujets décrit le voisinage spécifique de chaque groupe (c.f. tableau 4). Le Degree in représente le nombre de liens reçus par un groupe. Dans notre exemple, il mesure le nombre de groupes faisant directement référence au groupe concerné. Ainsi, «Un monde d'avance» est le groupe auquel les autres groupes font le plus référence (6 liens).

\begin{tabular}{|l|l|l|}
\hline & \multicolumn{1}{|c|}{ Degree in } & \multicolumn{1}{c|}{ Degree out } \\
\hline BH 2008 & 4 & 1 \\
\hline Mondedavan & 6 & 1 \\
\hline JCH & 1 & 2 \\
\hline Mondeda78 & 0 & 2 \\
\hline Mondeda34 & 0 & 2 \\
\hline Mondeda92 & 0 & 2 \\
\hline Mondedamarne & 0 & 1 \\
\hline
\end{tabular}

Tableau 4 : analyse du voisinage 
Néanmoins, ce groupe n'interagit que très peu avec les autres groupes (une liaison) ce qui montre qu'il y a un manque de réciprocité entre les groupes favorables à Benoît Hamon. Le réseau est dit peu agrégé et perd, de ce fait, en force et en efficacité de persuasion (manque de partage d'informations, de construction collaborative, d'échanges d'idées...).

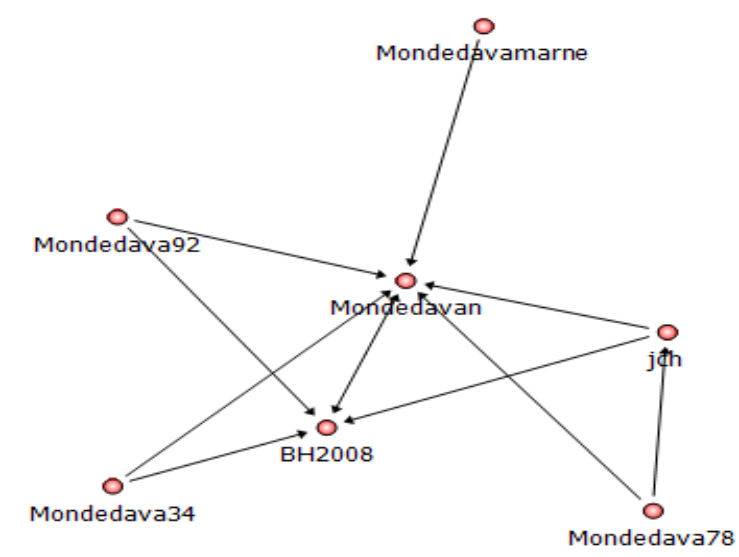

\section{Schéma 5 : centralité de proximité du groupe}

Ainsi, comme nous l'avons précédemment souligné, l'ARS décrit avec précision la centralité des membres d'une communauté virtuelle (Schéma 5). Cette centralité peut être analysée de façon longitudinale. Mazzoni E. (2006) s'appuyant sur Lave J. et Wenger E. (1991) insiste sur le fait que tout nouveau membre occupe d'abord une position périphérique d'observateur. Peu à peu l'intensification des échanges avec les autres membres du groupe lui permettra d'acquérir une position plus centrale. Néanmoins, cette légitimation de la place du membre au sein du groupe ne peut se construire que sur le partage d'informations et l'interactivité que ce dernier apporte (construction de connaissances, diffusion d'information...). Dans le cas du réseau politique de Benoît Hamon, le groupe «Un monde d'avance » dispose d'une centralité de pouvoir (Bonacich P. , 1987 cité par Mazzoni E. (2006)) car les connexions des autres groupes passent par lui et en sont donc dépendants. Par contre, le seul lien mutuel observé est celui enregistré avec « Benoît Hamon 2008 ».

\begin{tabular}{|l|l|} 
Indicateur de centralité de pouvoir (Degree in/Degree in + Degree out $)$ \\
\hline BH 2008 & 0,18 \\
\hline Mondedavan & 0,27 \\
\hline JCH & 0,04 \\
\hline Mondeda78 & 0 \\
\hline Mondeda34 & 0 \\
\hline Mondeda92 & 0 \\
\hline Mondedamarne & 0 \\
\hline
\end{tabular}

Tableau 5 : centralité de pouvoir 


\begin{tabular}{|lcc|}
\hline Distribution & \multicolumn{2}{c|}{ Valeurs } \\
\cline { 2 - 3 } & \multicolumn{1}{c|}{ Degree in } & Degree out \\
\hline Somme & 11 & 11 \\
Moyenne & 1,571 & 1,571 \\
Standard déviation & 2,259 & 0,495 \\
Minimum & 0 & 1 \\
Maximum & 6 & 2 \\
\hline$\%$ de membres isolés & 0 & \\
Inclusion (\%) & $100 \%$ & \\
\hline Nombre de dyades & Mutuelles : 1 & \\
& Asymétriques : 9 & \\
& Nulles : 11 & \\
& Score de réciprocité : 0,1 & \\
\hline
\end{tabular}

Tableau 6 : qualité des relations au sein du réseau

\subsection{Qualité de la production : groupes supporters et démocratie locale}

L'analyse des réseaux sociaux permet d'évaluer les rapports de force entre membres, les jeux de pouvoir, les formes optimales de circulation de l'information. Elle s'intéresse également à la qualité des productions des réseaux. Cette qualité passe par un certain nombre d'indicateurs qui vont de la taille des groupes à la contribution des membres (messages, éléments publiés...) ou à la valence des groupes (supporters ou opposants). La seconde étude réalisée porte sur les réseaux des candidates Ségolène Royal et Martine Aubry. 70 groupes ont été analysés constituant les groupes les plus récents référencés par Facebook et portant sur les deux personnalités. La composition des groupes indique que ceux-ci regroupent des supporters mais également des adversaires des deux candidates. La valence calculée montre que $29,3 \%$ des groupes étudiés sont favorables à Ségolène Royal contre 50\% pour Martine Aubry. Le profil des groupes hostiles montre que ces derniers ont une production et une richesse multimédia inférieures aux groupes de supporters. Par contre, il n'y a pas de différence significative entre les deux types de groupes concernant leur capacité à se diffuser au sein du réseau (taille du groupe, nombre d'amis de l'administrateur...) et leur interactivité (nombre de messages mur, nombre de participants aux forums de discussion).

\begin{tabular}{|l|l|l|l|}
\hline & Groupes supporters & Groupes opposants & Test de moyenne \\
\hline $\begin{array}{l}\text { Groupes associés } \\
\text { politiques }\end{array}$ & 2,7 & 0,7 & $\begin{array}{l}\text { F : 9,868 } \\
\text { Sig }: 0,002\end{array}$ \\
\hline Evénements & 1,11 & 0,02 & $\begin{array}{l}\text { F : 22,424 } \\
\text { Sig }: 0,000\end{array}$ \\
\hline $\begin{array}{l}\text { Forums discussions } \\
\text { nombre de sujets }\end{array}$ & 6,76 & 1,6 & $\begin{array}{l}\text { F : 4,952 } \\
\text { Sig }: 0,029\end{array}$ \\
\hline Forums discussion & 9,8 & 2,6 & F $: 5,001$ \\
\hline
\end{tabular}




\begin{tabular}{|l|l|l|l|}
\hline nombre d'éléments & & & Sig : 0,029 \\
\hline Photos & 12,1 & 4,2 & $\begin{array}{l}\text { F }: 7,218 \\
\text { Sig }: 0,009\end{array}$ \\
\hline Vidéos & 4,5 & 2,7 & $\begin{array}{l}\text { F : } 10,769 \\
\text { Sig }: 0,002\end{array}$ \\
\hline Publications & 8,5 & 0 & $\begin{array}{l}\text { F }: 7,421 \\
\text { Sig }: 0,008\end{array}$ \\
\hline
\end{tabular}

Tableau 7 : profil des groupes hostiles

Ainsi, les groupes hostiles se construisent autour d'une contestation peu argumentée. Leur affiliation politique est également moins marquée que chez les groupes de supporters. La production d'idées y est plus faible. Par ailleurs, les types de groupe pratiquant le plus l'interactivité au sein des membres sont les groupes de la catégorie «Art, sport et loisirs» suivis de la catégorie « Organismes, organisations politiques » $(\mathrm{F}(5 ; 68)=2,279 \mathrm{p}<.005)$. Ce résultat semble conforter les observations sur la vivacité de la démocratie locale au sein des réseaux. En effet, ce type de groupe se constitue autour de projets liés au quotidien des électeurs. Nous citerons les groupes catégorisés «Art, sport et loisirs » soutenant Martine Aubry et ses projets de développement d'infrastructures sportives comme le grand stade de Lille. Enfin, il apparaît que ce n'est pas la capacité informationnelle du groupe qui induit de l'interactivité mais bien sa faculté relationnelle. Nous rappelons que nous mesurons la production au nombre de forums de discussion ouverts. Cet indice est un signe de vivacité de la construction de la connaissance au sein d'une communauté virtuelle (Fata A., 2004). Par contre, la capacité du groupe à diffuser à l'extérieur influe essentiellement sur la richesse multimédia du groupe (photos, vidéos en ligne) puisqu'un groupe de taille importante a la possibilité de récupérer des éléments de toute nature ou de les concevoir (vidéos essentiellement). En outre, la réalisation d'une ACP (analyse en composantes principales) permet de positionner les groupes sur deux dimensions. Nous qualifierons la première dimension de récupération de l'information externe. Cette dimension est constituée par la variable «capacités informationnelles ». La seconde dimension concerne la transformation/ utilisation de cette information au sein du groupe. Elle regroupe les variables de richesse multimédia, diffusion, production, interactivité.

\begin{tabular}{|l|l|l|}
\hline Composante 1 & Composante 2 & Matrice des composants \\
\hline 0,922 & & Production \\
\hline 0,908 & & Messages Mur \\
\hline 0,877 & & Nombre de participants forums de discussion \\
\hline 0,805 & & Diffusion \\
\hline & 0,908 & Capacités informationnelles \\
\hline $79,5 \%$ & Couverture cumulée \\
\hline
\end{tabular}

Tableau 8 : matrice des composantes 


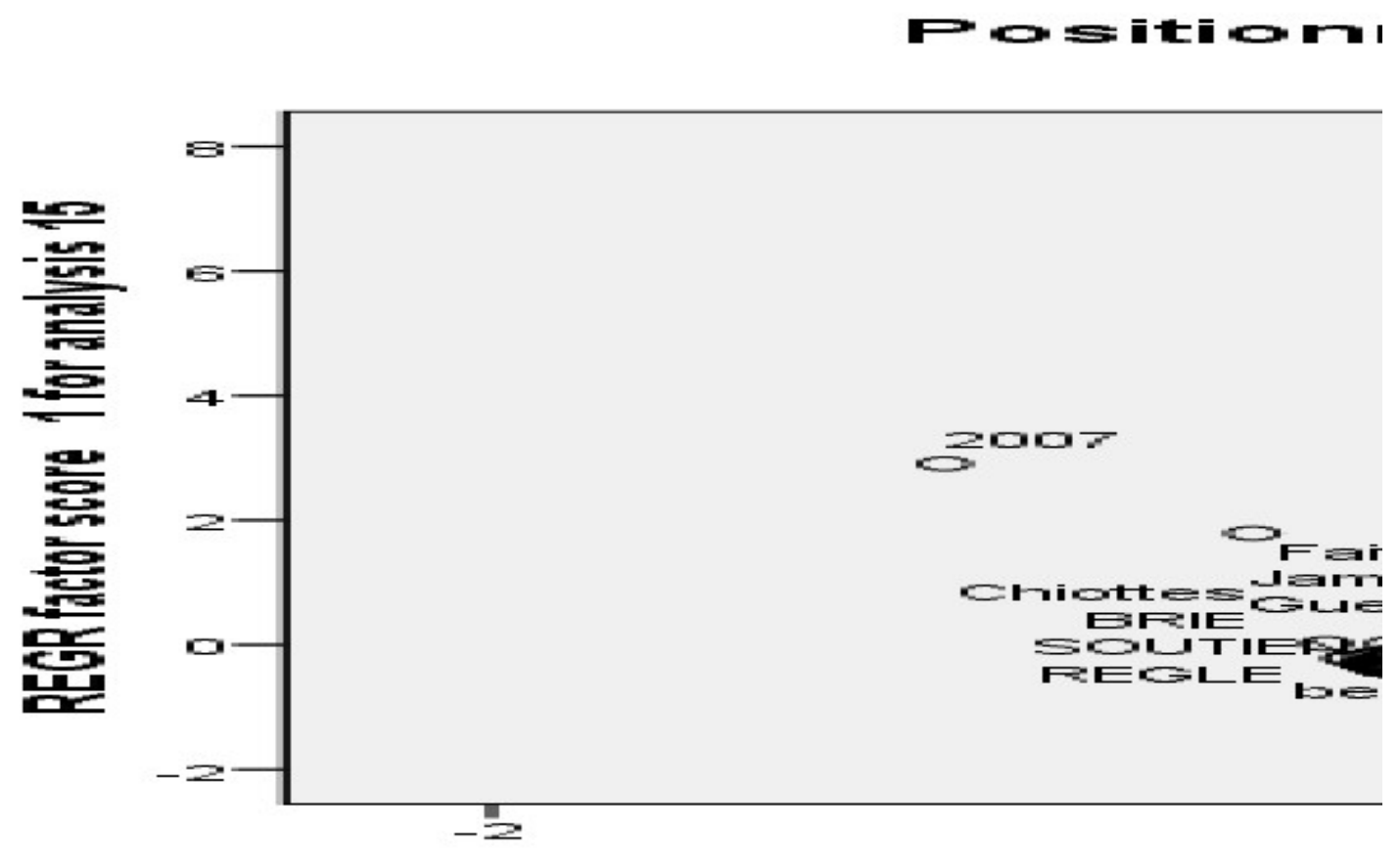

Schéma 6 : positionnement des groupes de l'échantillon

Dans la majorité des groupes, on observe une position équilibrée entre information restituée et connaissance produite. Seuls quelques groupes ont un positionnement distinctif. Les groupes « Stade» ou « Paris » mettent en ligne beaucoup d'informations extérieures. Ces groupes se sont créés autour de grands projets régionaux et tirent leur succès de la proximité développée avec les internautes. Le groupe « Echangin » a une production plus forte que les informations extérieures qu'il capte. Ce groupe est un groupe hostile et peut donc être considéré comme un outlier parmi les groupes hostiles, il devrait faire l'objet d'une surveillance particulière de la part des administrateurs. Les groupes de supporters à forte capacité informationnelle et à forte capacité de production doivent être privilégiés (renforcement de leur centralité) puisqu'ils constituent un pivot informationnel non négligeable. Néanmoins, aucun groupe n'est à la fois bien positionné sur l'informationnel et le relationnel ce qui pose question du lien pouvant exister entre mise en ligne d'une information et forte interactivité. Cette constatation illustre la difficulté d'animer un groupe qui est à la fois un média et un forum de discussion et d'interactivité. 


\section{DISCUSSION ET CONCLUSION}

Les résultats de ces deux études confortent un certain nombre d'enseignements issus de la littérature. Concernant l'analyse structurelle des réseaux, Granovetter M. (1973) montre qu'un réseau ouvert avec plusieurs liens faibles permet un accès à une quantité d'informations supplémentaires. La force des liens faibles est représentée par la structure du réseau de Benoît Hamon dans lesquels les groupes le supportant appartiennent eux-mêmes à une variété de réseaux qui n'est pas uniquement politique. Ces groupes issus du monde associatif, du sport, des regroupements d'étudiants constituent des ponts entre l'univers politique et les « autres mondes virtuels ». De ce fait, cette structure permet d'augmenter les trous structurels tels qu'ils ont été conceptualisés par Burt R.S. ${ }^{13}$ (1992). Enfin, elle apporte une réflexion modernisée sur la notion d'affinité et de ciblage. Mise en avant par Degenne A. et Forse M. (1994), la remise en cause de la catégorie au profit de la liaison revêt toute son importance en marketing politique. Ainsi, l'allocation des moyens doit se faire non pas en visant une catégorie définie (hommes, femmes, jeunes) mais une structure au sein de laquelle chaque membre n'a pas la même position. L'importance de certains groupes disposant d'une forte centralité de pouvoir doit être prise en compte dans la stratégie de persuasion élaborée par les partis. Au sein du même groupe peuvent se regrouper des individus très différents en terme de CSP ou d'âge. Néanmoins, la métastructure qui les réunit doit être considérée comme une des pierres angulaires possibles du développement du réseau. L'étude des dyades ou des triades permet de s'interroger sur la gestion de son réseau et la nécessité de créer des mutualisations ou d'éviter l'exclusion de groupes importants. Enfin, l'ARS permet une analyse dynamique des réseaux et de leurs évolutions. Le suivi des groupes périphériques destinés à devenir «groupe central» est d'autant plus important que la centralité est un élément de crédibilisation des discours. La maîtrise de l'information des groupes centraux considérée comme plus crédible que celle délivrée par les groupes périphériques a été démontrée par Cho H. et al. (2002).

\footnotetext{
${ }^{13}$ Caplow T. (1968) parle du troisième larron, celui qui est en relation avec deux acteurs qui ne sont pas en relation l'un avec l'autre. Ainsi, un trou structural désigne la séparation entre deux contacts non redondants (Mercklé P., 2004).
} 


\subsection{Apports théoriques et méthodologiques}

Un apport original de cette recherche réside dans l'intégration des groupes hostiles à l'analyse d'un réseau. Cette notion d'hostilité est peu développée dans la littérature. Nous montrons que des groupes peuvent se constituer uniquement sur l'opposition. La place de ces groupes au sein de sites de réseaux sociaux comme Facebook n'est pas à négliger. Elle peut « polluer » l'espace social d'un candidat et gêner le développement des groupes de soutien. Leur surveillance doit passer par une analyse de leur centralité afin d'évaluer leur pouvoir de nuisance. De même la notion de capital social assimilable à la richesse d'un réseau trouve son pendant dans le nombre de groupes hostiles, un candidat présentant un fort capital social peut parallèlement déclencher un opposition à la mesure de ses soutiens (c.f. Ségolène Royal). La politique sur le Web apparaît comme une nouvelle forme de sociabilité. Elle produirait du lien social et représenterait une nouvelle forme de sociabilité. La démarche volontaire d'appartenir à un groupe politique est révélatrice de l'implication au sein d'un réseau et plus généralement de l'affirmation d'une identité virtuelle. Elle remet en avant les opinions politiques comme faisant partie intégrante de l'identité d'un individu. D'un point de vue conceptuel, l'ARS remet en cause la notion d'espace public tel qu'il est appréhendé par les théoriciens du marketing politique. Achache G. (1989) présente l'espace public comme un lieu hors de la sphère des besoins et de la sphère de la vie privée et de l'intimité. Il serait également vu comme homogène et continu alors que les notions de diversité et de discontinuités (temps forts, croissance ou au contraire décroissance rapide des réseaux) sont à la base même de la «netpolitique ». Quant à la vie privée et l'intimité, elles se mêlent de façon inextricable aux opinions publiques. Enfin, il est possible de mener à partir de l'analyse des réseaux sociaux une réflexion sur une approche structurale du pouvoir. Moins les réseaux de contacts sont interconnectés entre eux, plus le capital social d'un candidat est important. Il multiplie en effet ses chances de diffusion de ses idées lorsque le réseau est large et faiblement redondant. Comme le souligne Mercklé P. (2004, p 65), «plus un acteur dispose de trous structuraux autour de lui, c'est-à-dire moins ses relations sont redondantes, moins elles se connaissent entre elles ou occupent des positions structuralement équivalentes, et plus il peut espérer tirer de son réseau des bénéfices importants ». Le capital social ne dépend pas du nombre de contacts, de la taille ou de l'ancienneté du réseau mais de sa forme. Cette forme doit privilégier les trous structuraux à savoir une maximisation des relations et une minimisation des connexions entre ces relations. D'un point de vue méthodologique, l'ARS 
apporte un enrichissement certain dans la gestion d'un réseau: identification des zones faibles, prise en compte et suivi des membres forts, quantification des interactions. Définie comme « une méthode de contextualisation des comportements » (Lazega E., 1998, p 116), l'ARS ne peut se suffire à elle-même sous peine de devenir une boîte à outils essentiellement descriptive de phénomènes dont l'observation doit être au service d'une théorie de l'action collective. De ce fait, elle nécessite un couplage avec d'autres méthodes d'analyse plus qualitatives (ethnographie) mais également quantitatives afin d'appréhender au mieux l'objet de recherche.

\subsection{Recommandations managériales}

Les recommandations managériales de cette recherche peuvent être synthétisés dans le tableau suivant :

\begin{tabular}{|l|}
\hline Consacrer du temps à visualiser son réseau, ses différentes régions et son évolution \\
\hline Identifier les groupes (favorables ou hostiles) bénéficiant d'une forte centralité \\
\hline Ne pas négliger les petits groupes \\
\hline $\begin{array}{l}\text { Disposer de la maîtrise d'un groupe à forte centralité comme tête de pont vers des groupes } \\
\text { similaires mais également n'appartenant pas à la catégorie «politique » }\end{array}$ \\
\hline $\begin{array}{l}\text { Ouvrir des groupes qui ne soient pas directement affiliés au candidat mais correspondent à } \\
\text { des grandes causes sociales et disposant d'une forte capacité de recrutement }\end{array}$ \\
\hline S'interroger sur la gestion des applications, créer des applications tierces \\
\hline $\begin{array}{l}\text { Ne pas faire de son groupe officiel un média classique mais plutôt un forum de discussion } \\
\text { interactif }\end{array}$ \\
\hline $\begin{array}{l}\text { Travailler sur les contenus visuels et audios, les renouveler et les enrichir de manière } \\
\text { régulière }\end{array}$ \\
\hline $\begin{array}{l}\text { Surveiller l'évolution des discours, l'observation in vivo des thématiques porteuses, des } \\
\text { faits marquants amenant un changement d'opinion }\end{array}$ \\
\hline $\begin{array}{l}\text { Encourager la viralité au sein même des groupes (amener les membres à poster sur leurs } \\
\text { profils les événements du candidat) }\end{array}$ \\
\hline $\begin{array}{l}\text { Entretenir de bonnes relations avec les administrateurs des groupes, récompenser leur } \\
\text { engagement }\end{array}$ \\
\hline $\begin{array}{l}\text { Ancrer ses groupes dans les vies locales (villes, régions, universités...) en développant des } \\
\text { synergies entre le virtuel (réseau) et la vie réelle (évènements, meetings) }\end{array}$ \\
\hline
\end{tabular}

\section{Tableau 9 : synthèse des recommandations managériales}

A l'instar des groupes créés de façon spontanée pour soutenir la candidature de Barack Obama, la gestion d'un réseau implique le recrutement d'administrateurs dédiés afin d'établir une véritable «stratégie réseau » en cohérence avec les programmes des candidats. 


\subsection{Voies futures de recherche}

La notion d'interactivité comme production collective de connaissance mériterait de plus amples développements. Mazzoni E. (2006) note «que la réalisation d'un bon réseau d'interactions entre les membres d'un groupe et surtout les réponses données aux messages reçus peuvent être les indicateurs d'une implication active et d'une participation dynamique aux activités collectives ». La production d'idées nouvelles issues de la base (démocratie participative) a trouvé Internet comme support privilégié. L'adjonction d'une dynamique de groupe en fait un enjeu majeur de l'enrichissement des discours des candidats. Nous avons montré qu'un petit groupe structuré peut produire plus d'interactivité entre ses membres qu'un grand groupe. Le rôle joué dans ce cadre par les administrateurs des groupes et leur influence comme leader d'opinion devraient être précisés. Cardon D. (2008) insiste sur l'individualisme démonstratif qui permet toutefois une coopération numérique forte. De même la notion d'interactivité serait dépendante du besoin de visibilité ce qui fait que les coopérations faibles peuvent déboucher sur de la coopération dans une seule logique opportuniste d'intérêt personnel (Cardon D., 2008). La gestion des administrateurs des groupes importants apparaît comme un élément incontournable de la réussite d'un réseau.

Le Buzz négatif comme l'a souligné Steyer A. et al. (2007) devrait être étudié sous l'angle de la diffusion des préférences car l'orientation positive ou négative des messages influence la diffusion du bouche à oreille et son efficacité. De nouvelles études pourraient également confirmer et comprendre les raisons de la faible production de contenus des groupes hostiles et de leur influence réelle sur la persuasion en matière politique. Il est à noter que leur manque d'argumentaires construits en fait pour les administrateurs réseaux des objets virtuels difficiles à circonscrire et à concurrencer sur le plan des idées. Enfin, les réseaux «petit monde » remettent en cause la loi des 20 -80 en amenant les spécialistes du marketing politique à s'interroger sur l'importance de l'émiettement des réseaux en une multitude de nombreux petits groupes. Qualifiées d'effet longue traîne, ces infinités de niche (Chabi S., 2008) correspondent aux régions moyennes observées dans notre étude empirique. L'optique « longue traîne » représente une voie de recherche prometteuse amenant à une redéfinition des critères de segmentation et de ciblage au-delà du monde politique. 


\section{REFERENCES BIBLIOGRAPHIQUES}

Achache G. (1989), Le marketing politique, éditions Hermès.

Barabasi A. et Albert R.A. (1999), Emergence of Scaling in Random Networks, Science, 286, 509-512.

Bennet W.L. (2003), Communicating global activism: strengths and vulnerabilities of network politics, Information, communication and society, 6(2), 143-168.

Bimber B. (1998), The Internet and political transformation: Populism, community and accelerated pluralism, Polity, XXXI(1), 133-160.

Bonacich P. (1987), Power and centrality: a family of measures, American Journal of Sociology, 92, 1170-1182.

Bott E. (1957), Family and Social Network, Londres, Tavistock.

Boyd D. M. et Ellison N. B. (2007), Social network sites: definition, history and scholarship, Journal of Computer-Mediated Communication, 13, 1.

Burt R. S. (1992), Structural holes: the social structure of competition, Cambridge, Harvard University Press.

Cai D., Shao Z., He X., Yan X. et Han J. (2005), Mining hidden community in heterogeneous social networks, Proceedings of the 3rd International Workshop on Link Discovery, ACM Press, Chicago, Illinois.

Calvani A., Fini A., Bonaiuti G. et Mazzoni E. (2005), Monitoring interactions in collaborative learning environments (CSCL): a tool kit for Synergeia, Journal of E-learning and Knowledge Society, 1, 63-73.

Caplow T. (1968), Two Against One,. Deux contre un. Les coalitions dans les triades, Paris, Armand Colin, 1971

Cardon D. (2008), le-design-de-la-visibilite-un-essai-de-typologie-du-web-2.0, Réseaux, 6, 152, 93-137.

Chabi S. (2008), De l'importance des réseaux sociaux en marketing, Reflets et perspectives de la vie économique, 2, XLVII, 95-102.

Cho H., Lee J.-S., Stefanone, M. et Gay G. (2005), Behaviour \& Information Technology, Nov- Dec 2005, 24, 6, 435-447.

Dahlberg L. (2007), Computer-mediated communication and the public sphere : a critical analysis, Journal of Computer-Mediated Communication, 7, 1. 
Degenne A. et Forse M. (1994), Les réseaux sociaux. Une approche structurale en sociologie; Paris, Armand Colin, collection U, 1994.

Dumoulin M. (2002), Les forums électroniques : délibératifs et démocratiques ? , Internet et la démocratie, Québec, Monière et Wollank éditeurs, 141-157.

Fata, A. (2004). Gli aspetti psicologici della formazione a distanza, Franco Angeli, Milano. Freeman L. C (1979), Centrality in social networks: conceptual clarification, Social Networks, $1,215-239$.

Füller J., Jawecki G. et Mühlbacher H. (2007), Innovation creation by online basketball. communities, Journal of Business Research, 60(1), 60-71.

Garton L., Haythornthwaite C. et B. Wellman (1997), Studying online social networks, Journal of Computer Mediated Communication, 3 (1).

Godes D. et Mayzlin D. (2004), Using Online Conversations to Study Word-of-Mouth Communication, Marketing Science, 23,4, 545-560.

Granovetter M. (1973), The strength of weak ties, American Journal of Sociology, 78, 13601380.

Greffet F.et Wojcik S. (2008), Parler politique en ligne. Une revue des travaux français et anglo-saxons, Réseaux, 4, 150, 19-50.

Haythornthwaite C. (2005), Social Networks and Internet Connectivity Effects, Information, Communication and Society, 8(2), 125-47.

Haythornthwaite C. (2005), Social Networks and Internet Connectivity Effects, Information, Communication and Society, 8(2), 125-47.

Iacobucci D. (2007), Let's Network!, Marketing Research, 19,1, 30-35.

Kumar R., Novak J. et Tomkins A. (2006), Structure and evolution of online social networks, Proceedings of the 12th ACM SIGKDD International Conference on Knowledge Discovery and Data Mining, 611-617.

Lave J. et Wenger E. (1991), Situated learning: legitimate peripheral participation, Cambridge, MA: Cambridge University Press.

Lazega E. (1998), Réseaux sociaux et structures relationnelles, Presses Universitaires de France, collection Que sais-je ?, Paris.

Lelong B. et Thomas F. (2001), L'apprentissage de l'internaute: socialiation et autonomisation, Actes du 3e Colloque International sur les Usages et Services des Télécommunications, Paris, 12-14

Matuszak C. (2007), Le forum comme lieu de production d'un discours unitaire et mobilisateur: un nouveau terrain pour la confrontation idéologique chez les mouvements 
politiques marginaux, $9^{\text {ème }}$ congrès de l'Association Française de Science Politique (AFSP), atelier 8, La discussion politique en ligne, enjeux théoriques et interrogations méthodologiques, Toulouse.

Mazzoni E. (2006), Du simple tracement des interactions à l'évaluation des rôles et des fonctions des membres d'une communauté en réseau : une proposition dérivée de l'analyse des réseaux sociaux, ISDM - Information Sciences for Decision Making, 25, 2006, 477-487. Mercklé P. (2004), Sociologie des réseaux sociaux, Repères, La Découverte, Paris.

Milgram S. (1967), The small world problem, Psychology Today, 1, 61-67.

Monnoyer-Smith L.(2006), How E-Voting Technology Challenges Traditional Concepts of Citizenship : an Analysis of French Voting Rituals ?, 2d International Workshop en Electronic Voting.

Putnam R.D.(2000), Bowling Alone. The Collapse and Revival of American Community, Simon \& Schuster.

Rechenmann J.-J. (2001) - Audit de site web, Paris, Editions d'Organisation.

Rivière C. (2000), La sociabilité téléphonique, Revue française de Sociologie, 41, 4, 685-717. Schneider S.M. (1996), Creating a Democratic Public Sphere Through Political Discussion : a Case Study of Abortion Conversation on the Internet, Social Science Computer Review, 14,4, 373-393.

Sing S. (2006), Social network and group formation, theorical concepts to leverage, www.boxesandarrows.com/view/social-networks.

Steyer A., Garcia-Bardidia R. et P. Quester (2007), Modélisation de la structure sociale des groupes de discussion sur Internet : implications pour le contrôle du marketing viral, Recherches et Applications en Marketing, 22, 3.

Williams C.B. et Gulati G.J. (2006), The evolution of online campaigning in congressional elections, Paper presented at the annual meeting of the American Political Science Association, Philadelphia. 